\title{
ZERO POINTS OF KILLING VECTOR FIELDS, GEODESIC ORBITS, CURVATURE, AND CUT LOCUS
}

BY

\author{
WALTER C. LYNGE
}

ABSTRACT. Let $(M, g)$ be a compact, connected, Riemannian manifold. Let $X$ be a Killing vector field on $M . f=g(X, X)$ is called the length function of $X$. Let $D$ denote the minimum of the distances from points to their cut loci on $M$. We derive an inequality involving $f$ which enables us to prove facts relating $D$, the zero ponts of $X$, orbits of $X$ which are closed geodesics, and, applying theorems of Klingenberg, the curvature of $M$. Then we use these results together with a further analysis of $f$ to describe the nature of a Killing vector field in a neighborhood of an isolated zero point.

1. An inequality.

Theorem 1. Let $X$ be a Killing vector field on M. Let $q$ be a critical point of the length function $f=g(X, X)$ of $X$ such that $f(q) \neq 0$. Assume the orbit $\gamma$ of $X$ through $q$ is closed. Let $a$ be another point of $M$ and suppose the distance from $q$ to $a$ is $\rho$. Then we have

$$
(\sqrt{f(q)}-\sqrt{f(a)}) \nu / \sqrt{f(q)} \leq 2 \rho .
$$

Proof. Denote by $\beta$ the period of the orbit $\gamma$. We note that $\gamma$ is a geodesic, since $q$ is a critical point of $f[2$, p. 356]. Let $\tau$ be the orbit of $X$ through $a$.

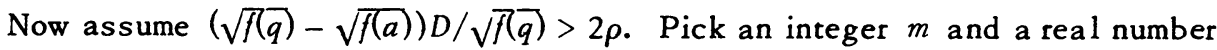
$r$ so that

$$
m \beta-r=D / \sqrt{f(q)}-\delta
$$

where $\delta>0$ is chosen sufficiently small so that $(\sqrt{f(q)}-\sqrt{f(a))(D / \sqrt{f(q)}}-\delta)$ $>2 \rho$. Then we have

$$
\sqrt{f(q)}(m \beta-r)-\sqrt{f(a)}(m \beta-r)>2 \rho .
$$

Let $d(v, w)$ denote the distance between two points $v$ and $w$ in $M$. Let $\phi_{t}$ be the flow of $X$. We have $\phi_{m \beta-r}(q)=\phi_{m \beta}\left(\phi_{-r}(q)\right)=\phi_{-r}(q)$ since $m$ is an integer and $\beta$ is the period of $\gamma$. The length of the shortest segment of $\gamma$ between $q$ and $\phi_{-r}(q)$ is $\int_{0}^{m \beta-r} \sqrt{f}\left(\phi_{t}(q)\right) d t$, which is equal to $\sqrt{f}(q)(m \beta-r)$ since $f$ is constant along $\gamma$. This is the length of the shortest segment because

Received by the editors December 13, 1971 .

AMS (MOS) subject classifications (1970). Primary 53B20, 53C20, 57D25. curvature.

Key words and phrases. Killing vector field, cut locus, geodesic orbit, sectional 
by (2), $\sqrt{f(q)}(m \beta-r)=D-\delta \sqrt{f(q)}<D$ and clearly $D \leq 1 / 2$ (length of $\gamma$ ). This is also the length of the shortest segment of $\gamma$ between $q$ and $\phi_{r}(q)$. Since $\gamma$ is a geodesic and since the length of the shortest segment of $\gamma$ between $q$ and $\phi_{r}(q)$ is less than $D$, this segment is therefore length minimizing. Hence we have

$$
d\left(q, \phi_{r}(q)\right)=\sqrt{f(q)}(m \beta-r) .
$$

Since $f$ is constant along the orbit $\tau$ of $X$ through $a$, we have that the length of a segment of $\tau$ between $\phi_{m} \beta^{(a)}$ and $\phi_{r}(a)$ is $\sqrt{f(a)}(m \beta-r)$. Thus we have

$$
d\left(\phi_{m \beta}(a), \phi_{r}(a)\right) \leq \sqrt{f(a)}(m \beta-r) .
$$

From (3), (4), and (5) we obtain $d\left(q, \phi_{r}(q)\right)>2 \rho+\sqrt{f(a)}(m \beta-r) \geq 2 \rho+$ $d\left(\phi_{n \beta}(a), \phi_{r}(a)\right)$. Hence

$$
d\left(q, \phi_{r}(q)\right)>2 \rho+d\left(\phi_{m \beta}(a), \phi_{r}(a)\right) .
$$

By the triangle inequality we obtain

$$
d\left(q, \phi_{r}(a)\right) \leq d\left(q, \phi_{m \beta}(a)\right)+d\left(\phi_{m \beta}(a), \phi_{r}(a)\right) .
$$

But $d\left(q, \phi_{m \beta}(a)\right)=d\left(\phi_{m \beta}(q), \phi_{m}(a)\right)=d(q, a)=\rho$ since $\phi_{m \beta}(q)=q$ and since $\phi_{m} \beta$ is an isometry. Thus

$$
d\left(q, \phi_{r}(a)\right) \leq \rho+d\left(\phi_{m \beta}(a), \phi_{r}(a)\right) .
$$

Since $\phi_{r}$ is an isometry, we have

$$
d\left(\phi_{r}(a), \phi_{r}(q)\right)=d(q, a)=\rho .
$$

We observe that (7) implies $\phi_{r}(a)$ lies in a closed ball about $\phi_{r}(q)$ of radius $\rho+d\left(\phi_{m} \beta(a), \phi_{r}(a)\right)$. And (8) implies that $\phi_{r}(a)$ lies in a closed ball about $\phi_{r}(q)$ of radius $\rho$. But by (6), these two balls have empty intersection. Hence we have that (1) is true.

From Theorem 1 we obtain immediately the following

Theorem 2. Suppose $X$ is a Killing vector field on $M$ and $q$ is a critical point of $f=g(X, X)$ such that $f(q) \neq 0$. Suppose the orbit of $X$ through $q$ is closed. If $p$ is a zero point of $X$, then $d(p, q) \geq D / 2$.

In particular, this theorem gives a lower bound for the distances between zero points of $X$ and orbits of $X$ which are nontrivial closed geodesics. Moreover, the lower bound depends only on the metric and not on the vector field $X$. To show that it cannot be improved, consider the following example: Let $M$ be $S^{2}$ with the usual metric. Let $X$ be the Killing vector field whose flow is a 1 parameter group of rotations about an axis through the north and south poles. Then any point $q$ on the equator is a critical point of $f=g(X, X)$ such that $f(q) \neq 0$ and the north pole $N$ is a zero point of $X . D$ in this case is half of the 
circumference of $S^{2}$. Thus we have $d(N, q)=D / 2$.

Under additional assumptions on $M$ we can give a lower bound in terms of curvature:

Theorem 3. Suppose $M$ is a compact, connected Riemannian manifold of even dimension. Suppose the sectional curvatures $K_{\sigma}$ satisfy $0<K_{\sigma} \leq \lambda$ for all tangent planes $\sigma$. Let $X$ be a Killing vector field on $M$. Then the distance from any zero point of $X$ to any orbit of $X$ which is a nontrivial closed geodesic is always $\geq \pi / 4 \sqrt{\lambda}$. If in addition we assume $M$ is orientable or simply connected (wbich are equivalent assumptions), then this distance is $\geq \pi / 2 \sqrt{\lambda}$.

Proof. By theorems of Klingenberg [1, pp. 227 and 230], $D \geq \pi / 2 \sqrt{\lambda}$ ( $D \geq \pi / \sqrt{\lambda}$ if $M$ is orientable). The result now follows immediately from Theorem 2 .

As a corollary of Theorem 2 we have the following criterion for the zero set of a Killing vector field to be empty in terms of the distribution throughout $M$ of orbits which are nontrivial closed geodesics.

Theorem 4. Suppose $X$ is a Killing vector field on $M$ with the property that for any point $a$ in $M$ there exists an orbit $\gamma_{a}$ of $X$ which is a nontrivial closed geodesic such that the distance from a to $\gamma_{a}$ is $<D / 2$. Then the zero set of $X$ is empty.

2. Isolated zero point of $X$. Let $X$ be a Killing vector field on $M$ with an isolated zero point at $p$. Then $p$ is a critical point of the function $f=g(X, X)$. We recall that to $f$ there is associated a symmetric bilinear functional $f_{* *}$ on $T_{p} M$ called the Hessian of $f$ at $p$. The index of $f$ at $p$ is the maximal dimension of a subspace of $T_{p} M$ on which $f_{* *}$ is negative definite. The critical point $p$ is nondegenerate if the nullity of $f_{* *}$ on $T_{p} M$ is zero.

Lemma. $p$ is a nondegenerate critical point of index zero of the function $f=g(X, X)$.

Proof. Since $X$ is Killing and $p$ is an isolated zero point, there exists [3, p. 64] a coordinate neighborhood $U$ of $p$ with local coordinates $\left\{x^{1}, \cdots, x^{n}\right\}$ such that (i) $x^{i}(p)=0, i=1, \cdots, n$, (ii) if $X=\sum_{i=1}^{n} \xi^{i}\left(\partial / \partial x^{i}\right)$ on $U$, then $\xi^{i}(p)=0$ and the matrix with $(i, j)$ entry $\left.\left(\partial \xi^{i} / \partial x^{j}\right)\right|_{p}$ is of the form

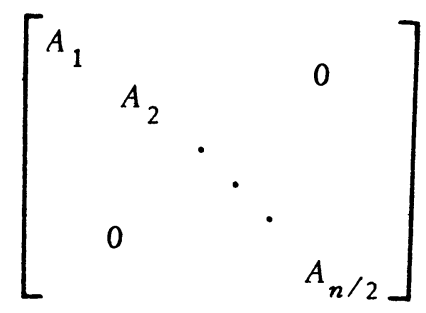


where

$$
A_{k}=\left[\begin{array}{cc}
0 & \theta_{k} \\
-\theta_{k} & 0
\end{array}\right], \quad \theta_{k} \neq 0, k=1, \cdots, n / 2
$$

and

(iii) if $g=\Sigma_{i, j} a_{i j} d x^{i} \otimes d x^{j}$, then $a_{i j}(p)=\delta_{i j}$. Thus we have $f=$ $\sum_{i, j=1}^{n} \xi^{i} \xi^{j} a_{i j}$

Let $v=\left.\Sigma_{i} v^{i}\left(\partial / \partial x^{i}\right)\right|_{p}$ and $w=\left.\Sigma_{i} w^{i}\left(\partial / \partial x^{i}\right)\right|_{p}$ be two vectors in $T_{p} M$. Extend these to vector fields $\widetilde{v}=\Sigma_{i} v^{i}\left(\partial / \partial x^{i}\right)$ and $\tilde{w}=\Sigma_{i} w^{i}\left(\partial / \partial x^{i}\right)$ on $U$. Then by definition $f_{* *}(v, w)=\tilde{v}_{p}(\widetilde{w}(f))$. By a direct computation we see that

$f_{* *}(v, w)=2\left\{\theta_{1}^{2}\left(v^{1} w^{1}+v^{2} w^{2}\right)+\theta_{2}^{2}\left(v^{3} w^{3}+v^{4} w^{4}\right)+\cdots+\theta_{n / 2}^{2}\left(v^{n-1} w^{n-1}+v^{n} u^{n}\right)\right\}$.

From this it is obvious that $f_{* *}$ is positive definite on all of $T_{p} M$ and the nullity of $f_{* *}$ is zero. Hence we have the result.

Theorem 5. There exists a local coordinate neighborbood $U$ of $p$ with local coordinates $\left\{x^{1}, \ldots, x^{n}\right\}$ such that with respect to the se coordinates, $f=\left(x^{1}\right)^{2}$ $+\cdots+\left(x^{n}\right)^{2}$ on $U$.

Proof. This is an immediate consequence of the previous lemma and the Morse lemma [4, p. 6].

We have that the following situation prevails near $p$ :

Theorem 6. There exists a connected open neighborbood $U$ of $p$ satisfying

(i) $U-\{p\}$ is a disjoint union of bypersurfaces of $M$, each of them diffeomorphic to $S^{n-1}$. ( $n$ (even) is the dimension of M.) We call these byperspheres.

(ii) The function $f$ is constant on each bypersphere.

(iii) $X$ is tangent to each bypersphere.

(iv) Restricted to each bypersphere, the length of $X$ is constant.

Proof. (i) and (ii) follow from Theorem 5. (iii) follows from the fact that $f$ is constant along each integral curve of $X$, and hence any integral curve of $X$ which meets $U-\{p\}$ lies in one of the hyperspheres. (iv) comes from (ii) and (iii).

Next we consider the question: How far can the neighborhood $U$ be extended s.) that the conditions of Theorem 6 continue to hold?

Theorem 7. There exists a connected open neighborbood $V$ of $p$ which contains the neighborbood $U$ of Theorem 6 and which also satisfies conditions (i)-(iv). Moreover, $\bar{V}$ meets an orbit of $X$ which is a nontrivial geodesic.

Proof. Let $Y$ be the vector field $\operatorname{grad} f /\|\operatorname{grad} f\|^{2}$ defined on the open submanifold $M-B$ of $M$, where $B$ denotes the set of critical points of $f . Y$ is of 
course nowhere zero on $M-B$. We remark that the orbits (as point sets) of $Y$ are the same as the orbits of $\left.\operatorname{grad} f\right|_{M-B}$. These orbits are perpendicular to the level surfaces of $f$. Let $r>0$ be sufficiently small so that the level surface $f=r$ has a nonempty intersection $S$ with the neighborhood $U$ of Theorem 5 . Then $S$ is a hypersphere. Let $t \rightarrow \alpha_{t}(v)$ be the integral curve of $Y$ through an arbitrary point $v$ of $S$ with $a_{0}(v)=v$. Then $d \alpha_{t}(v) / d t=\operatorname{grad} f /\|\operatorname{grad} f\|^{2}$. Hence we have

$$
\frac{d f\left(\alpha_{t}(v)\right)}{d t}=g\left(\operatorname{grad} f, \frac{\operatorname{grad} f}{\|\operatorname{grad} f\|^{2}}\right)=1 .
$$

Thus we have that

$$
f\left(\alpha_{t}(v)\right)=f\left(\alpha_{0}(v)\right)+t=r+t
$$

and $a_{t}(v)$ is defined for $t$ in an interval $\left(-r, \epsilon_{v}\right)$. Let $\epsilon=\inf { }_{v \in S^{\epsilon}}$. Then there exists a family of local diffeomorphisms $\left\{\phi_{t}\right\}_{t \in(-r, \epsilon)}$, each defined on a neighborhood of $S$. For $v$ in $S, \phi_{t}(v)=\alpha_{t}(v)$. Thus $f\left(\phi_{t}(v)\right)=r+t$ for any $v$ in $S$ by (1). Hence $f$ has the constant value $r+t$ on $\phi_{t}(s)$ and, since $\phi_{t}$ is a diffeomorphism, $\phi_{t}(s)$ is a hypersphere. Now let $V=\{p\} \cup \bigcup_{t \in(-r, \epsilon)} \phi_{t}(s)$. Then $V \supseteq U$ and $V$ satisfies conditions (i)-(iv).

Now we show that $\bar{V}-\{p\} \cap B \neq \varnothing$. For suppose the contrary. Let $A$ be the open ball about $p$ whose boundary is $S_{z}$ Let $v$ be an arbitrary point of $S$. Consider the curve $[0, \epsilon) \rightarrow M: t \rightarrow a_{t}(\nu)$. This curve is contained in the compact set $\bar{V}-A \subset M-B$. We have that $\|Y\|$ is bounded from above on this compact set. This means that the length of the curve $[0, \epsilon) \rightarrow M: t \rightarrow a_{t}(v)$ is finite, where the length of this curve is defined by $\lim _{t \rightarrow \epsilon} \int_{0}^{t}\left\|Y\left(\alpha_{s}(v)\right)\right\| d s$. It then follows easily that $\lim _{t \rightarrow \epsilon} \alpha_{t}(v)$ exists. Call this limit $p_{v}$. Then $p_{v}$ is in $\bar{V}-A$.

Let $\rho$ be the distance from $B$ to $\bar{V}-A$. It is clear that the integral curve of $Y$ starting at $p_{v}$ goes on for a distance (measured along the integral curve) of at least $\rho / 2$. Now let $C=\{q \in M \mid d(q, \bar{V}-A) \leq \rho / 2\}$. Then $C$ is compact and $C \subset M-B$. Thus $\|Y\|$ is bounded from above on $C$. This implies there exists $\eta>0$ such that the curve $[0, \epsilon) \rightarrow M: t \rightarrow \alpha_{t}(\nu)$ may be extended to $[0, \epsilon+\eta) \rightarrow$ $M: t \rightarrow \alpha_{t}(v)$ and $\eta$ is independent of $v$ in $S$. This contradicts the definition of $\epsilon$. Thus $\bar{V}-\{p\} \cap B \neq \varnothing$. From the construction of $V$ it is obvious that if $q$ is a point in $\bar{V}-\{p\} \cap B$, then $f(q) \neq 0$. Thus the orbit of $\dot{X}$ through $q$ is a nontrivial geodesic meeting $V$. This concludes the proof.

To show that in general the neighborhood $V$ cannot be further extended in such a way that conditions (i)-(iv) still hold, we need only consider the projective plane $w$ ith the Killing vector field induced by the vector field $X$ on $S^{2}$ given in the example after Theorem 2. 
According to Theorem 7 , it is reasonable to regard the distance $d$ from $p$ to the nearest orbit of $X$ which is a nontrivial geodesic as a measure of the "size" of the neighborhood $V$. In case all the orbits of $X$ are closed, we have by Theorem 2 a lower bound for $d$ which is independent of $X$ : namely, $d \geq D / 2$. Moreover, a manifold having a Killing vector field with an isolated zero point is even dimensional [3, p. 63]. Thus if the sectional curvatures $K_{\sigma}$ of $M$ satisfy $0<K_{\sigma} \leq \lambda$, we have by Theorem 3 that $d \geq \pi / 4 \sqrt{\lambda}$, again assuming all orbits of $X$ are closed.

\section{BIBLIOGRAPHY}

1. D. Gromoll, W. Klingenberg and W. Meyer, Riemannsche Geometrie im Grossen, Lecture Notes in Math., no. 55, Springer-Verlag, Berlin and New York, 1968. MR 37 $\# 4751$.

2. Robert Hermann, Differential geometry and the calculus of variations, Math. in Sci. and Engineering, vol. 49, Academic Press, New York, 1968. MR 38 \#1635.

3. Shôshichi Kobayashi, Fixed points of isometries, Nagoya Math J. 13 (1958), 63-68. MR $21 \# 2276$.

4. J. Milnor, Morse theory, Ann. of Math. Studies, no. 51, Princeton Univ. Press, Princeton, N. J., 1963. MR $29 \# 634$.

DEPARTMENT OF MATHEMATICS, EDGECLIFF COLLEGE, CINCINNATI, OHIO 45206

Current address: Department of Mathematics, Northern Kentucky State College, Highland Heights, Kentucky 41076 